\title{
Experimental and Numerical Investigation of Swirling Flow on Triple Elbow Pipe Layout
}

\author{
Hideharu Takahashi', San Shwin², Ari Hamdani', Nobuyuki Fujisawa3 ${ }^{3}$, Hiroshige Kikura1 \\ ${ }^{1}$ Laboratory for Advanced Nuclear Energy, Institute of Innovative Research, Tokyo Institute of Technology, Tokyo, Japan \\ ${ }^{2}$ Graduate School of Science and Engineering, Tokyo Institute of Technology, Tokyo, Japan \\ ${ }^{3}$ Department of Mechanical Engineering, Niigata University, Niigata, Japan \\ Email: htakahashi@lane.iir.titech.ac.jp
}

How to cite this paper: Takahashi, H., Shwin, S., Hamdani, A., Fujisawa, N. and Kikura, H. (2020) Experimental and Numerical Investigation of Swirling Flow on Triple Elbow Pipe Layout. Journal of Flow Control, Measurement \& Visualization, 8, 45-62.

https://doi.org/10.4236/jfcmv.2020.82003

Received: October 18, 2019

Accepted: December 8, 2019

Published: April 30, 2020

Copyright (c) 2020 by author(s) and Scientific Research Publishing Inc. This work is licensed under the Creative Commons Attribution International License (CC BY 4.0).

http://creativecommons.org/licenses/by/4.0/

\section{(c) (i) Open Access}

\begin{abstract}
The secondary flow downstream of a triple elbow layout was studied experimentally and numerically to visualize the flow behavior under swirling inlet flow conditions. The inlet swirling condition was generated by a swirl generator, consisting of a rotary pipe and honeycomb assembly. The experiments were carried out in turbulent water flow condition at Reynolds number $\mathrm{Re}=$ $1 \times 10^{4}$ and inlet swirl intensity $S=1$. Ultrasonic measurements were taken at four locations downstream of the third elbow. The two-dimensional velocity field of the flow field was measured using the phased array ultrasonic velocity profiler technique to evaluate the flow field with separation. Furthermore, a numerical simulation was performed and its results were compared with the experimental data. The numerical result was obtained by solving three-dimensional, Reynolds-averaged Navier-Stokes equations with the renormalization group $k-\varepsilon$ turbulence model. The experimental results confirmed that the swirling flow condition modified the size of the separation region downstream of the third elbow. A qualitative comparison between the experimental and CFD simulation results of the averaged velocity field downstream of the third elbow showed similar tendency on reverse flow.
\end{abstract}

\section{Keywords}

Swirling Flow, Triple Elbow, Ultrasound, Phased Array Sensor, Velocity Profile, CFD, Numerical Simulation

\section{Introduction}

Turbulent flow through a compact piping system is encountered in a variety of industrial applications and is used in power plants. Complicated flow condition 
occurs in the region downstream of the compact pipe layout, where secondary swirling flow can be generated downstream of the elbow. Investigations into velocity fluctuations in elbow pipe layouts remain challenging in the study of industrial fluid dynamics. Under swirling flow condition, significant pressure fluctuation and high-velocity fluctuation occur in the elbow. These are sources of flow-induced vibration, which is a crucial problem in industrial piping systems [1] [2] [3] [4]. Furthermore, swirling flow enhances flow accelerated corrosion of the pipe wall, thereby causing pipe break accidents in the piping systems of power plants [5] [6] [7]. Hence, investigations into flow structure and velocity fluctuation are essential for pipeline safety management.

A multiple elbow pipe layout can be found in the loop system, i.e., cold-leg piping, of the Japan Sodium-cooled Fast Reactor (JSFR). The cold-leg piping in the JSFR has three successive short elbows. Owing to its complexity, Ebara et al. [8] [9] studied the flow field and pressure fluctuation in the third elbow of a triple elbow piping with small curvature radius using $1 / 7$-scale models of the cold-leg piping of the JSFR.

In previous studies, computational fluid dynamics (CFD) simulation using $\mathrm{OpenFOAM}^{\circ}$ on a $90^{\circ}$ elbow was performed to characterize the swirling secondary flow downstream of an elbow in a pipe [10]. A comparative study was performed on the selection of turbulence models for analysis. One of the first studies was carried out by Al-Rafai et al. [11], who provided the flow structure through an elbow. They performed experiments on a turbulent flow at Reynolds number $\mathrm{Re}=3.4 \times 10^{4}$ in two types of elbows (pipe diameter to elbow radius ratio $\gamma=0.07$ and 0.14 ) using Laser Doppler Velocimetry (LDV) and compared the results with those obtained from the numerical simulations using the $k-\varepsilon$ model. They showed the distributions of mean and root mean square velocities in the elbows. The results indicated that secondary flow is magnified in the elbow with increasing pipe diameter to elbow radius ratio $\gamma$. Hilgenstock and Ernst [12] tested two well-known turbulence models ( $k-\mathcal{E}$ model and renormalization group model known as RNG) and provided acceptable results. An experimental study of turbulent flow in an elbow with imposed swirl was carried out by Kalpakli and Örlü [13]. They used particle image velocimetry (PIV) to study the formation of Dean vortices and swirl motion at values of swirl intensity $S$ in the range $0-1.2, S$ being defined as the ratio of circumferential momentum to axial momentum, in the downstream region of an elbow. Chang and Lee [14] also investigated the effects of swirl on the secondary flow field along an elbow at Re values in the range $(1.0-2.5) \times 10^{4}$. In these studies, LDV and PIV were applied to investigate the mean swirling velocity fields and flow structures. However, these systems are limited to flow through transparent pipes owing to optical access, and hence, it is difficult to apply these techniques in the case of actual plant pipes. Therefore, a non-invasive measurement technique should be applied to evaluate the velocity field and velocity fluctuation in an elbow pipe. In this study, an ultrasonic technique is applied to measure the two-dimensional veloc- 
ity field downstream of a triple elbow pipe layout.

Takeda [15] developed an Ultrasonic Velocity Profiler (UVP) to measure instantaneous velocity profiles of non-transparent media and opaque liquid flows. Initially, the conventional UVP method is employed to measure the one-dimensional velocity profile in the measurement line. Two-dimensional velocity vector measurements, Takeda and Kikura [16] investigated the velocity field of mercury flow using the UVP system with multiple transducers. Nevertheless, the measurement system using multiple transducers becomes quite complicated as the number of transducers increases. A phased array UVP system was developed to minimize the effects of this problem [17]. A phased array sensor has multiple ultrasonic piezoelectric elements; an ultrasound beam can be steered to a specific angle by controlling the time delay of ultrasound transmission from each piezoelectric element. Therefore, multiple lines can be measured, and the two-dimensional velocity field can be evaluated by using a single phased array sensor. The performance of phased array UVP in mapping velocity flow was confirmed by Fukumoto et al. [17] for the detection of water leakage from a tank.

In this study, the flow fields downstream of a triple elbow layout were studied experimentally using phased array UVP and numerically using ANSYS ${ }^{\oplus}$ Fluent v16.2. The objective of this study is to visualize the flow behavior downstream of the third elbow and to clarify the flow separation region. The influence of an inlet swirling condition on flow separation is presented and discussed. In addition, a numerical simulation was performed to validate the Reynolds-averaged Navier-Stokes (RANS) model in the case of flow downstream of the triple elbow layout.

\section{Experimental Investigation}

\subsection{Measurement Principle and Hardware System}

The working principle of the phased array UVP system is based on Doppler shift frequency detection along ultrasound beam lines. The phased array sensor emits an ultrasonic pulse, and each element of the sensor receives the echo reflected from the surface of a particle. The exciting element emits a spherical ultrasonic wave. When adjacent elements emit waves within a second of each other, interference of wave fronts occurs, as shown in Figure 1(a). A schematic of a phased array sensor is shown in Figure 1(b). The interference pattern depends on the time delay so that the steering angle of the ultrasonic beam can be changed by the time delay $\Delta t$. The steering angle $\theta_{s}$ is related to the time delay $\Delta t$, speed of sound $c$, and inter-element spacing $d$, and the relationship is expressed as the following equation:

$$
\theta_{s}=\sin ^{-1}\left[\frac{c \Delta t}{d}\right]
$$

The Doppler shift frequency $f_{D i}$ is observed at each element using the following equation: 


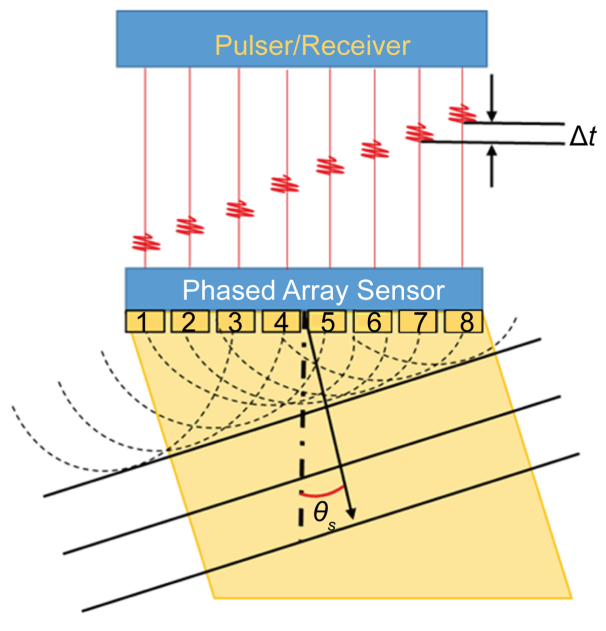

(a)

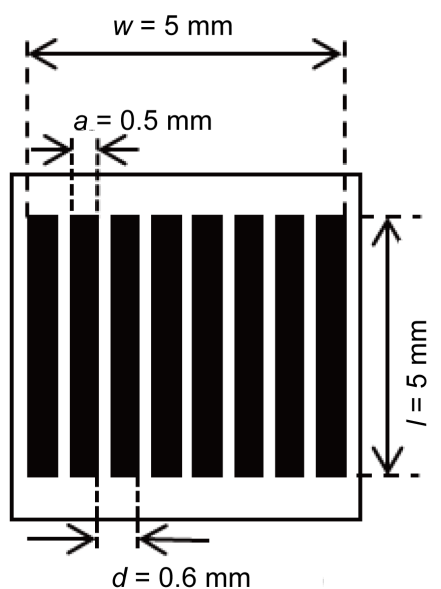

(b)

Figure 1. Beam steering principle of phased array sensor and its schematic. (a) Beam steering; (b) Schematic of a phased array sensor.

$$
f_{D i}=\frac{f_{0}}{c}\left(\boldsymbol{e}_{e}+\boldsymbol{e}_{i}\right) \cdot \boldsymbol{V}
$$

where the Doppler shift frequency is observed at the $\mathrm{i}^{\text {th }}$-channel element, $\boldsymbol{e}_{e}$ is the unit vector in the direction of the measurement line, $\boldsymbol{e}_{i}$ is the unit vector in the direction from the particle to the $\mathrm{i}^{\text {th }}$-channel element, $c$ is the speed of sound in water, $f_{0}$ is a basic frequency of the phased array sensor, and $\boldsymbol{V}$ is the particle velocity. Equation (3) shows that the Doppler shift frequency received at each element depends on the positions of the elements. The first and eighth elements, which are shown in Figure 2, are considered to receive an echo signal each from the particle, and the velocity vector can be reconstructed from the first and eighth elements using Equation (4).

$$
\begin{gathered}
{\left[\begin{array}{l}
f_{D 1} \\
f_{D 8}
\end{array}\right]=\frac{f_{0}}{c}\left[\begin{array}{l}
\boldsymbol{e}_{e}+\boldsymbol{e}_{1} \\
\boldsymbol{e}_{e}+\boldsymbol{e}_{8}
\end{array}\right]} \\
\boldsymbol{V}=\frac{c}{f_{0}}\left[\begin{array}{l}
\boldsymbol{e}_{e}+\boldsymbol{e}_{1} \\
\boldsymbol{e}_{e}+\boldsymbol{e}_{8}
\end{array}\right]^{-1} \cdot\left[\begin{array}{l}
f_{D 1} \\
f_{D 8}
\end{array}\right]
\end{gathered}
$$

The phased array UVP hardware system used for the two-dimensional velocity field measurement is shown in Figure 3. The National Instrument LabVIEW program was used to control the phased array UVP system and reconstructed the measured velocity into a two-dimensional velocity vector field. The measurement system consists of a $2 \mathrm{MHz}$ phased array sensor with eight piezoelectric elements, an eight-channel pulse receiver, an analog to digital converter and personal computer. The computer was also used to control the pulse receiver and analyzed the echo signal from the digital converter.

\subsection{Experimental Set-up}

The experiment was conducted in a water circulation system, which consists of a cooling system and electromagnetic flow meter as shown in Figure 4(a). Nylon 


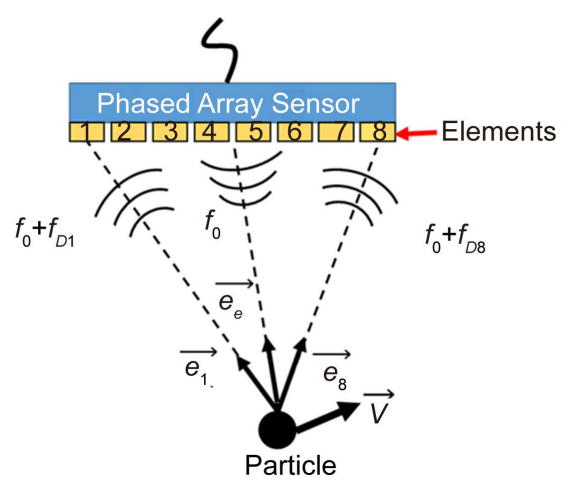

Figure 2. Velocity vector reconstruction of a moving particle.

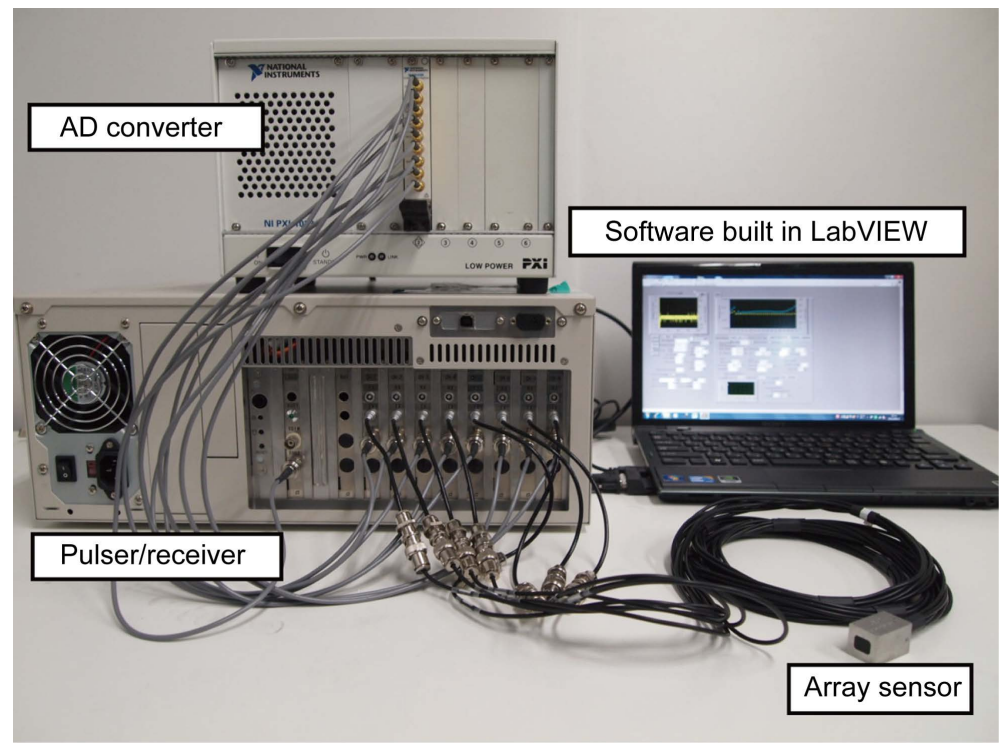

Figure 3. A system for two-dimensional velocity measurement (Phased Array UVP).

particles (WS200P) with mean diameter and density of $80 \mu \mathrm{m}$ and $1.02 \mathrm{~g} / \mathrm{cm}^{3}$, respectively, were used as ultrasonic reflectors because the density was nearly equal to that of working water. The particles were added to the working water to achieve a concentration of $0.1 \mathrm{~g} / \mathrm{L}$. The inner diameter of the pipe was $D=50$ $\mathrm{mm}$ and the pipe was made of acrylic resin. The axial distance from the inlet to the triple elbow pipe layout was $42 D$. Therefore, the inlet velocity profile of the flow was that of a fully developed turbulent pipe flow condition. The elbow had a radius of curvature $R=25 \mathrm{~mm}$. Thus, the pipe diameter to elbow radius ratio $\gamma=D / R=2$. The present triple elbow pipe layout was a $1 / 17$ scale model of the JSFR, while the Reynolds number of the flow was $\mathrm{Re}=1 \times 10^{4}$. The cooling system controlled the water temperature to $25^{\circ} \mathrm{C} \pm 1^{\circ} \mathrm{C}$, which was the same as that of the surrounding environment. Temperature measurements were carried out using a thermocouple during the experiments.

Several experimental methods have been proposed to generate swirling flow, 


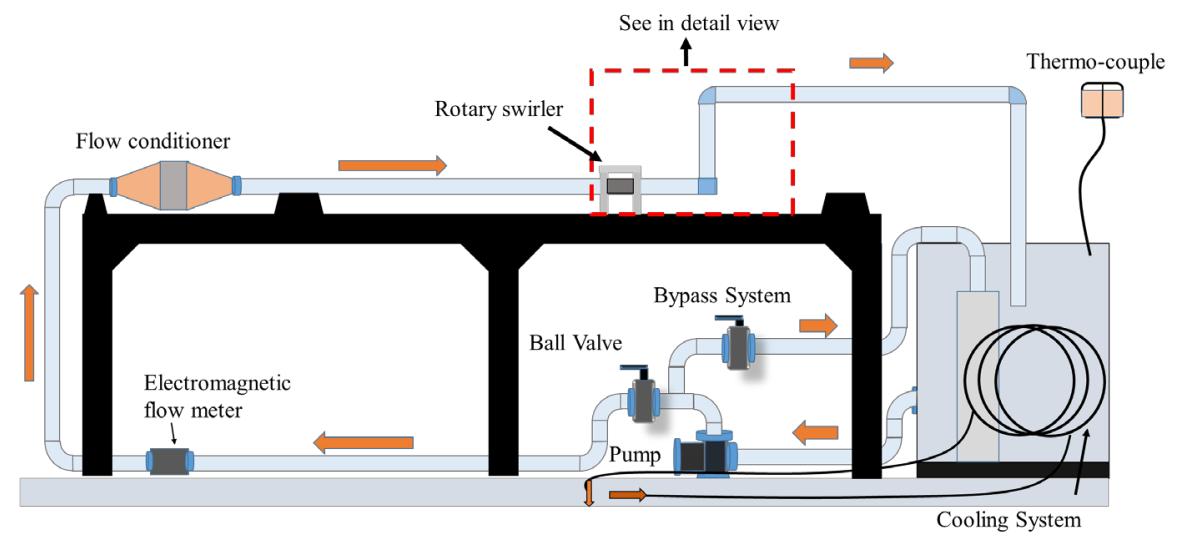

(a)

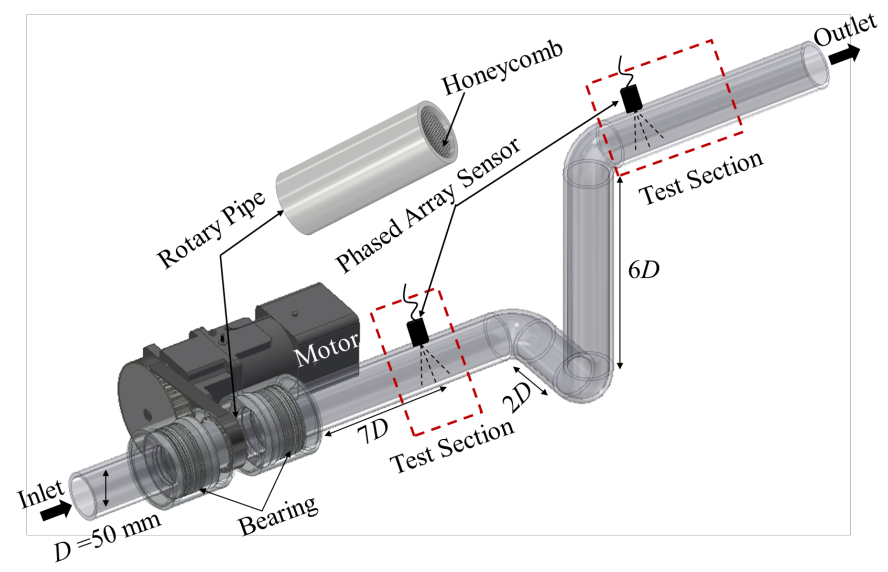

(b)

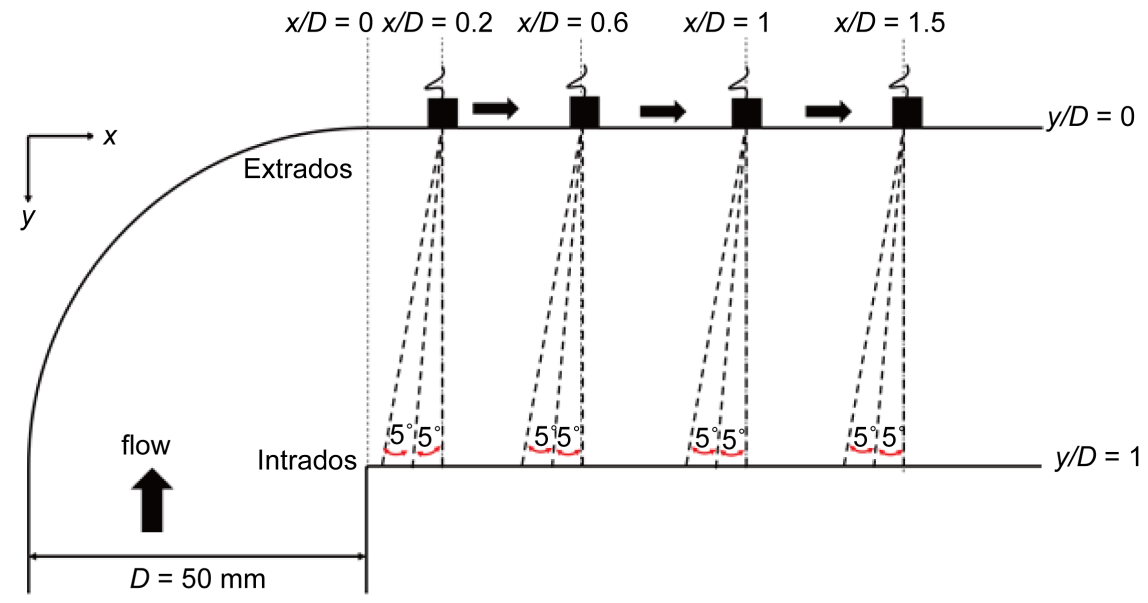

(c)

Figure 4. Schematic view of the experimental setup. (a) Experimental facilities of a piping system; (b) Detail view of the swirl generator and pipe layout; (c) Test section downstream of the third elbow.

such as rotary pipe, tangential flow injection, and vanes. They have different effects on the velocity distribution of swirling flow [18]. In this study, a rotary pipe was used as the swirl generator [7]. The swirl generator was installed at a dis- 
tance of $10 D$ upstream of the triple elbow pipe layout. A detailed view of the experimental set up with the swirl generator is shown in Figure 4(b). A honeycomb was inserted into the swirl generator to generate axisymmetric swirling flow. This means that the swirling core downstream of the swirl generator was located in the center of the pipe. The swirl intensity could be easily controlled by changing the rotating rate of the swirl generator. The swirl intensity can be evaluated from the following equation under the assumption that the viscous effect is negligible [7]:

$$
S=\frac{\int_{0}^{R} U_{z} U_{\theta} r^{2} \mathrm{~d} r}{R \int_{0}^{R} U_{z}^{2} r \mathrm{~d} r}=\frac{1}{2} R \omega / U
$$

where $r$ is the radial distance from the pipe axis, $U_{z}$ is the mean axial velocity, and $U_{\theta}$ is the mean circumferential velocity. Equation (5) shows that the swirl intensity can be evaluated directly from the angular velocity $\omega$ of the swirl generator when the radius $R$ of the pipe and bulk velocity $U$ of the flow through the pipe is given.

A phased array sensor, with a basic frequency of $2 \mathrm{MHz}$, was installed on the pipe wall. Thus, there was direct contact between the sensor and water to overcome refraction in the pipe wall and water. The axial, radial and circumferential velocity profiles of the inlet swirling flow were measured at a distance of $7 D$ downstream of the swirl generator. The inlet velocity profile measured was used for the inlet boundary conditions of the CFD simulation. Table 1 shows a summary of the experimental conditions.

Table 1. Experimental conditions.

\begin{tabular}{cc} 
Parameters & Value \\
\hline Reynolds number $\left[\operatorname{Re}=\rho D U_{m} / \mu\right]$ & 10,000 \\
Dean number $\left[\mathrm{De}=\operatorname{Re}\left(R_{c} / D\right)^{0.5}\right]$ & 7,071 \\
Fluid (water) temperature & $25^{\circ} \mathrm{C} \pm 1^{\circ} \mathrm{C}$ \\
Angular speed of swirl generator $\omega$ & min: $0 \mathrm{~min}^{-1}$; max: $480 \mathrm{~min}^{-1}$ \\
Swirl intensity $S$ & $0 ; 0.25 ; 0.5 ; 0.75$; and 1 \\
Frequency of phased array transducer & $2 \mathrm{MHz}$ \\
Steering angle $\theta_{s}$ & $0 ;-5 ;$ and $-10^{\circ}$ \\
Pulse repetition frequency $f_{\text {prf }}$ & $1 \mathrm{kHz}$ \\
Number of repetition $N_{\text {rep }}$ & 256 \\
Spatial resolution $\Delta y$ & $0.74 \mathrm{~mm}$ \\
Time resolution $\Delta t\left[N_{\text {rep }} / f_{p r f}\right]$ & $0.256 \mathrm{~s}$ \\
Number of velocity profiles & 10,000 \\
\hline
\end{tabular}


For the axial flow measurements, there were four measurement positions at $x / D=0.2,0.6,1$, and 1.5 downstream of the third elbow to evaluate the velocity fluctuation in the secondary flow. After the measurement was conducted at the first position, i.e., $x / D=0.2$, the measurements were taken at other positions by moving the phased array sensor to $x / D=0.6, x / D=1$ and $x / D=1.5$. At each measurement positions, the steering angle of the ultrasonic beam was set to $0^{\circ}$, $-5^{\circ}$, and $-10^{\circ}$. As a result, three measurement lines were obtained at $5^{\circ}$ intervals (see Figure 4(c)).

The ultrasonic velocity profiler (UVP-Duo) was used to measure the one-dimensional instantaneous velocity downstream of the third elbow. The range of Reynolds number Re was 10,000 - 30,000 and that of swirl intensity $S$ was 0.25 - 1.0. Figure 5(a) and Figure 5(b) show the experimental setup and detailed view of the test section, respectively, for one-dimensional velocity measurement.

\section{Numerical Simulation}

A CFD analysis was performed for the turbulent flow through the triple elbow pipe layout. The model domain and mesh were established using the design

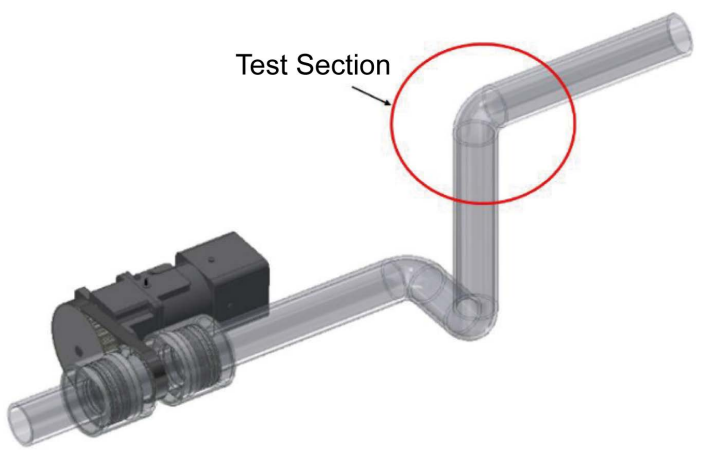

(a)

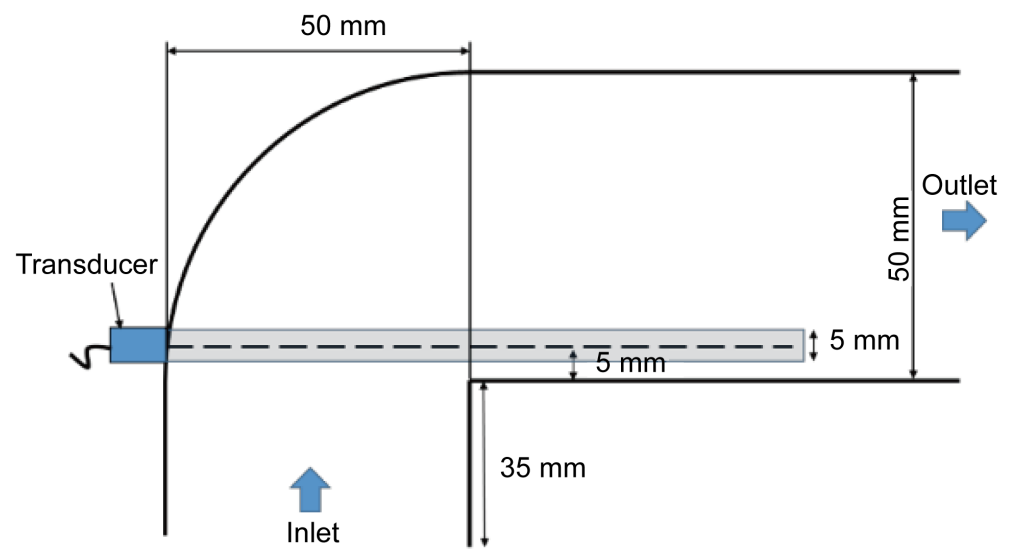

(b)

Figure 5. Schematic view of the experimental setup of UVP-Duo (one-dimensional velocity). (a) Test section of third elbow for one-dimensional velocity measurement; (b) Detailed view of the test section for one-dimensional velocity measurement. 
modeler and meshing tool in ANSYS $^{\circledast}$ software as shown in Figure 6. The CFD code ANSYS $^{\circledast}$ Fluent (version 16.2) was used to simulate pipe flow through a triple elbow pipe layout as shown in Figure 6(a). The inlet length upstream of the triple elbow was $3 D$, which was shorter than the experimental inlet length, because the swirl generator was not modeled in the numerical simulation. Therefore, the experimental data on swirling velocities were directly used as the inlet boundary conditions of the numerical simulation. The pipe length from downstream of the second elbow to the third elbow was set to $6 D$ which was similar to the experimental condition. The mesh type was a polyhedral cell, and the total number of meshes was approximately 1.4 million. A polyhedral cell was used to obtain the proper orthogonal and skewness values. The mesh near the pipe wall had an inflection layer of thickness $0.02 \mathrm{~mm}$ which corresponds to the non-dimensional wall distance $y+<1$ and the total number of layer was seven. The mesh quality of the outlet cross-sectional plane is shown in Figure 6(b).

The RNG $k-\varepsilon$ model was used to obtain a converged solution considering calculation time and cost. The RNG $k-\varepsilon$ model is preferable for the secondary flow and swirling flow conditions [19]. It is noted that the RNG model has an additional term in its $\varepsilon$ equation that significantly improves its accuracy for rapidly

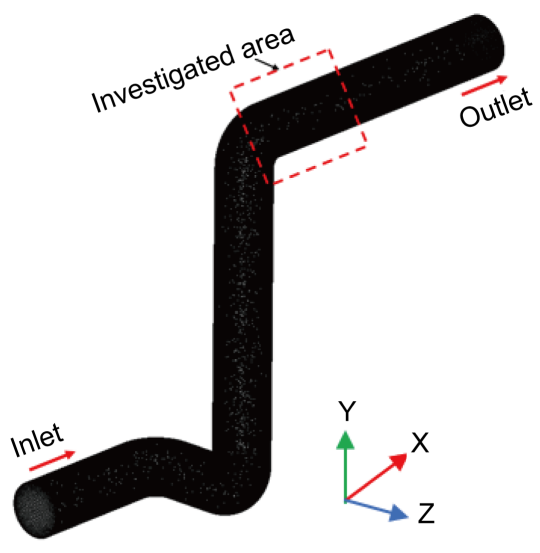

(a)

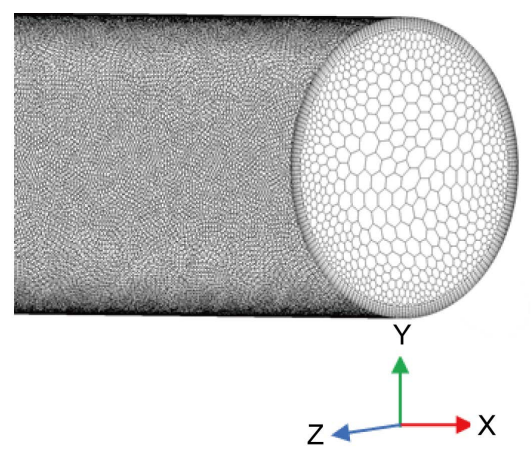

(b)

Figure 6. Schematic view of pipe geometry and polyhedral mesh quality. (a) Simulation pipe geometry; (b) Detailed view of polyhedral mesh quality. 
strained flows (i.e., elbow pipe). A previous study by Kim et al. [3] showed that the RNG $k-\mathcal{E}$ model was in good agreement with experimental results for swirling flow in a $90^{\circ}$ elbow. The non-equilibrium wall function was applied near the wall. The SIMPLE scheme was used as a coupling scheme between velocity and pressure. The PRESTO! scheme was used for spatial discretization of pressure, and the Quick scheme was applied for other spatial discretization. Table 2 shows the numerical conditions for the calculation of turbulent flow.

The initial condition of turbulent kinetic energy $(k)$ and turbulent dissipation rate $(\varepsilon)$ in the CFD simulation were evaluated from the following equations [19]:

$$
\begin{gathered}
k=\frac{3}{2}(I \cdot \bar{u})^{2} \\
\varepsilon=\frac{C_{\mu} \cdot k^{3 / 2}}{l}
\end{gathered}
$$

where $C_{\mu}=0.0845$ and $l$ is turbulent length scale,

$$
l=0.07 d_{h}
$$

$I$ is turbulent intensity which is defined as follows;

$$
I=0.16 \mathrm{Re}^{-1 / 8}
$$

where $d_{h}$ is hydraulic diameter (pipe diameter). The boundary conditions for velocity and pressure were prescribed by the inlet and outlet, respectively. The inlet swirling velocity profiles were imported from the experiment. It is noted that a thermal analysis was not included in the calculation, because the working water temperature was the same as room temperature. The outlet boundary condition was outflow with a static pressure equal to $0 \mathrm{~Pa}$, and the turbulence kinetic energy $(k)$ and dissipation rate $(\varepsilon)$ were set to $1 \mathrm{~m}^{2} / \mathrm{s}^{2}$ and $1 \mathrm{~m}^{2} / \mathrm{s}^{3}$ respectively.

Table 2. Numerical simulation conditions.

\begin{tabular}{cc}
\hline Parameters & Values \\
Turbulence model & $k-\mathcal{\varepsilon}$ (RNG) \\
Time & Steady state \\
Pressure-velocity coupling & SIMPLE \\
Gradient & Least squares cell based \\
Pressure & PRESTO! \\
Momentum & Quick \\
Turbulent kinetic energy & Quick \\
Specific dissipation rate & Quick \\
Mesh type & Polyhedral cell \\
Number of meshes & $1.4 \times 10^{6}$ \\
Inlet velocity & Axial, radial and tangential velocity profiles from \\
& the experiment
\end{tabular}




\section{Results and Discussion}

\subsection{Axisymmetric Swirling Flow}

The axial, radial and tangential velocities of the swirling flow were measured by using a phased array sensor at a distance of $7 D$ downstream of the swirl generator. The sensor position was converted into the axial and cross-sectional planes to measure all the axial, radial and circumferential velocities. Subsequently, these data were used for the inlet boundary conditions of the CFD simulation.

Figure 7 shows the radial distributions of the axial, radial and tangential velocities of swirling flow under the conditions of swirl intensity $S=1$ and Reynolds number $\operatorname{Re}=1 \times 10^{4}$. Owing to the axisymmetric nature of the velocity

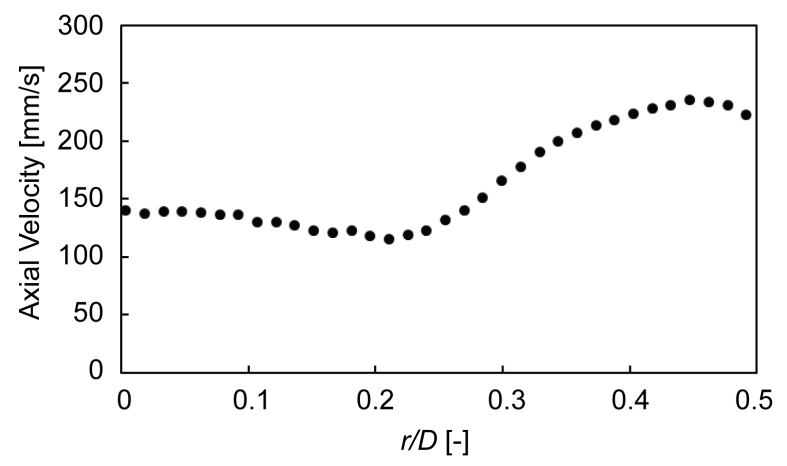

(a)

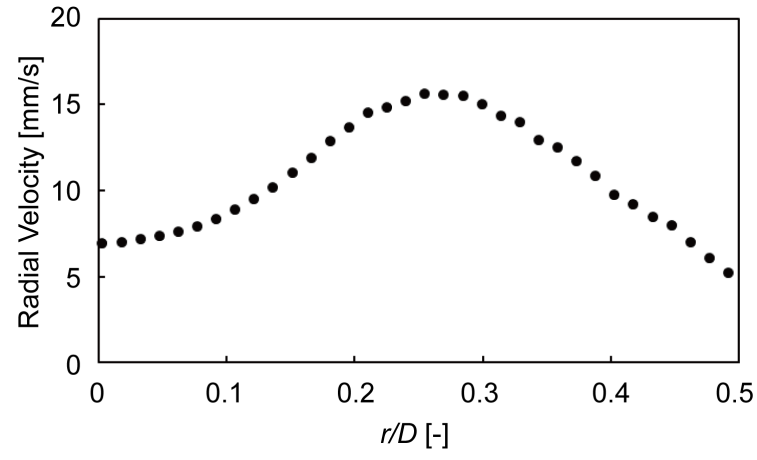

(b)

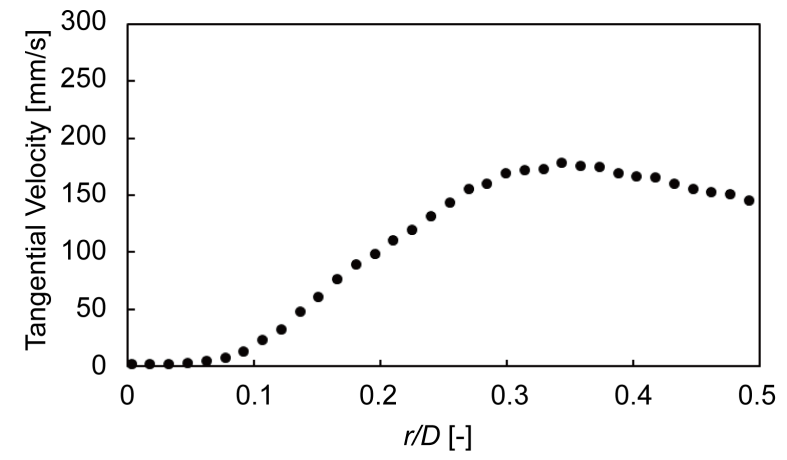

(c)

Figure 7. Experimental results of axial, radial and circumferential swirling flow velocities ( $S=1, \operatorname{Re}=1 \times 10^{4}$ ). (a) Axial velocity; (b) Radial velocity; (c) Tangential velocity. 
profiles, half velocity profiles are shown in the figure. The asymmetry of the velocity fields is experimentally confirmed by the measurements using the phased array UVP, shown in Figure 8. In Figure 7, $r / D=0$ is the center of the pipe and $r / D=0.5$ is on the pipe wall. The mean axial velocity of swirling flow is lower near the pipe center than in the outer layer of the swirling flow, while the tangential velocity is higher in the outer layer of the swirling flow than near the pipe center. The axial and tangential velocities do not approach zero near the wall because of the lower spatial resolution of UVP measurement. It is noted that the spatial resolution of UVP very close to the wall is $0.74 \mathrm{~mm}$. The radial velocity is much smaller than other velocity magnitudes. The flat nature of the axial velocity profile near the pipe center comes from the low level of swirl intensity in the pipe flow.

\subsection{Comparison between Experimental Results and CFD Simulation}

Two-dimensional velocity fields are measured to visualize the secondary flow downstream of the third elbow. A time average velocity profile was obtained by averaging 10,000 velocity profiles.

Figure 9 shows the measurement results at $x / D=0.2$ to $x / D=1.5$ downstream of the triple elbow pipe layout. The vertical axis indicates the dimensionless distance of the measurement line through the pipe. The horizontal axis is the axial measurement position downstream of the triple elbow. The result at $x / D=$ 0.2 shows the occurrence of reverse flow near the sharp edge elbow, which indicates the starts of flow separation near the sharp edge of the elbow (i.e., separation point). The separated flow reattaches near the first measurement line at $x / D$ $=1$ (i.e., reattachment point).Thus, the recirculating flow region is located between the separation point and reattachment point $(0.2<x / D<1)$ and the secondary reverse flow occurs at $0.7<y / D<1$ in the recirculating flow region. This means that there is no back flow downstream of the reattachment point $(x / D>$ 1). However, the accelerated swirling flow remains still active beyond the reattachment point because of the large velocity magnitude in the outer layer of the

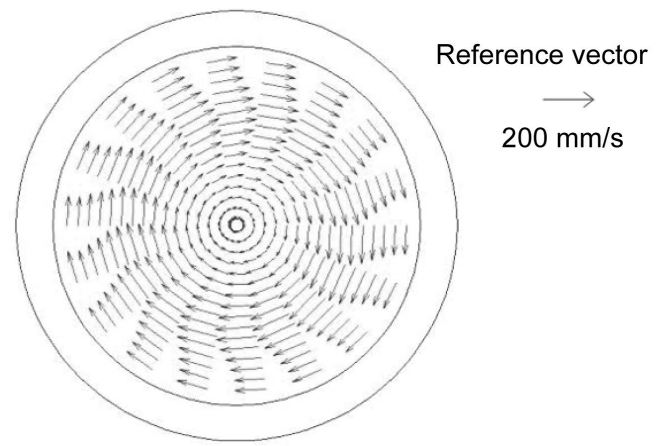

Figure 8. Time-averaged velocity vector fields at a distance of $7 D$ downstream of the swirl generator $\left(S=1, \operatorname{Re}=1 \times 10^{4}\right)$. 


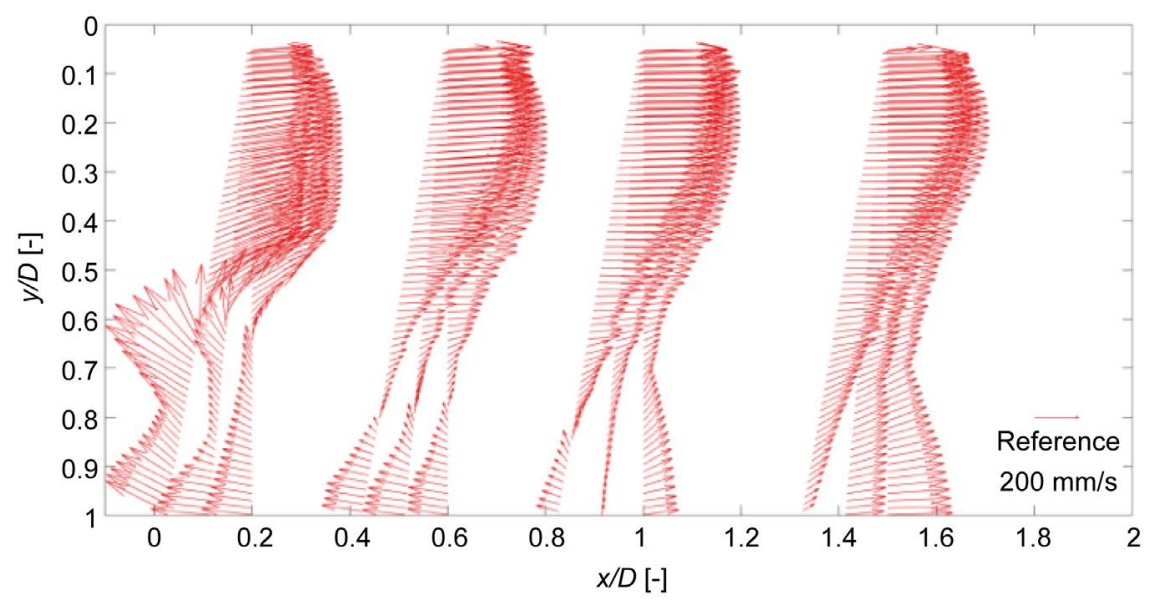

Figure 9. Two-dimensional time average axial velocity from experiment.

pipe flow.

Figure 10 shows the two-dimensional velocity field of the swirling flow from the numerical simulation. The axial, radial and circumferential velocity profiles of the swirling flow in the experimental data were used as the inlet boundary conditions, and the geometry of pipe layout was the same as that in the experimental setup. The experimental and numerical results showed similar tendency on reverse flow, however, further study will be needed for the difference of their detailed flow patterns. The velocity magnitude of the reverse flow in the numerical simulation is underestimated near the separation point, i.e., at around $x / D=$ 0.2. This is mainly owing to anisotropic turbulence near the flow separation. However, isotropic turbulence is assumed in the RANS model in the CFD simulation. Therefore, in the flow separation, the reverse flow velocity in the numerical simulation is underestimated compared to the experimental result.

The reattachment point in the numerical simulation is similar to the experimental result, i.e., at around $x / D=1$. This means that the area of the reverse flow region is well reproduced in the numerical simulation. The flow visualization of the numerical simulation in the cross-sectional plane is plotted to observe the detailed flow field of the secondary flow. Thus, we can consider that the experimental flow field is well reproduced by the numerical simulation.

The velocity contour is studied by visualizing the cross-sectional planes to investigate the velocity distribution downstream of the triple elbow pipe layout. Figures 11 (a)-(d) shows the numerical results at $x / D=0.2,0.6,1$ and 1.5 , respectively. The top of each figure represents the outside of the elbow (extrados), and the bottom is the inside of the elbow (intrados). We can observe the slow reverse flow in the cross-sectional velocity distribution near the intrados of the elbow (see Figure 11(a) and Figure 11(b)). Two counter-rotating vortex cells (Dean vortices), which are caused by the centrifugal force, can be recognized near the left and right regions of the elbow cross-section.

The flow pattern changes gradually as the flow advances downstream (Figures 11 (c) and Figure $11(\mathrm{~d})$ ). At $x / D=1$, the counter-rotating vortices are weakened 


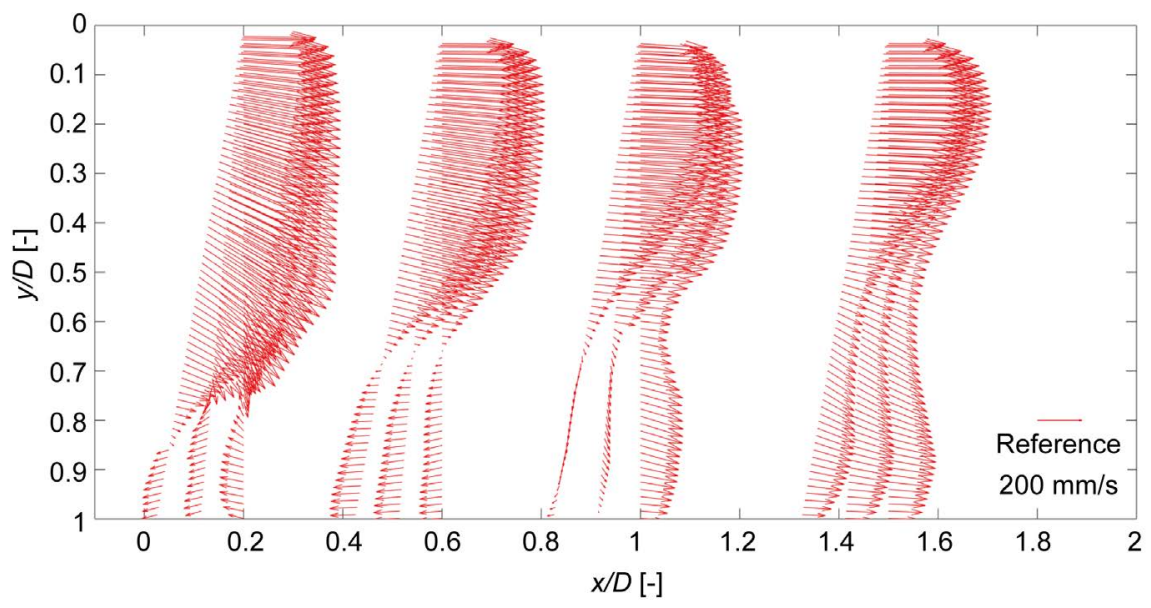

Figure 10. Two-dimensional time average axial velocity from numerical simulation.

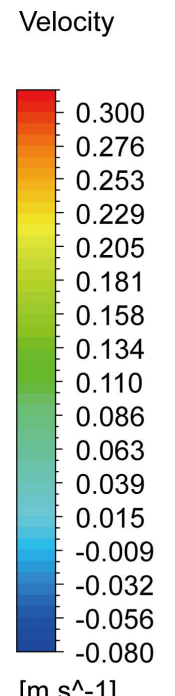

[m s^-1]

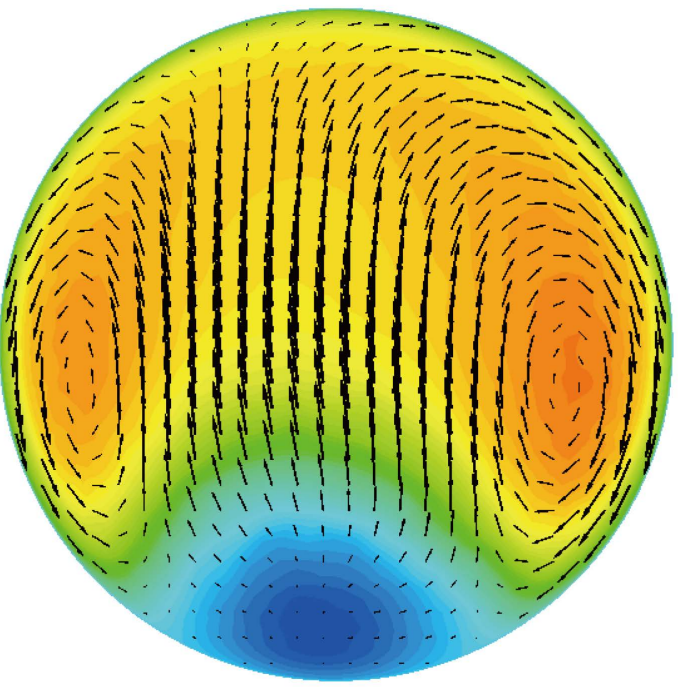

(a)

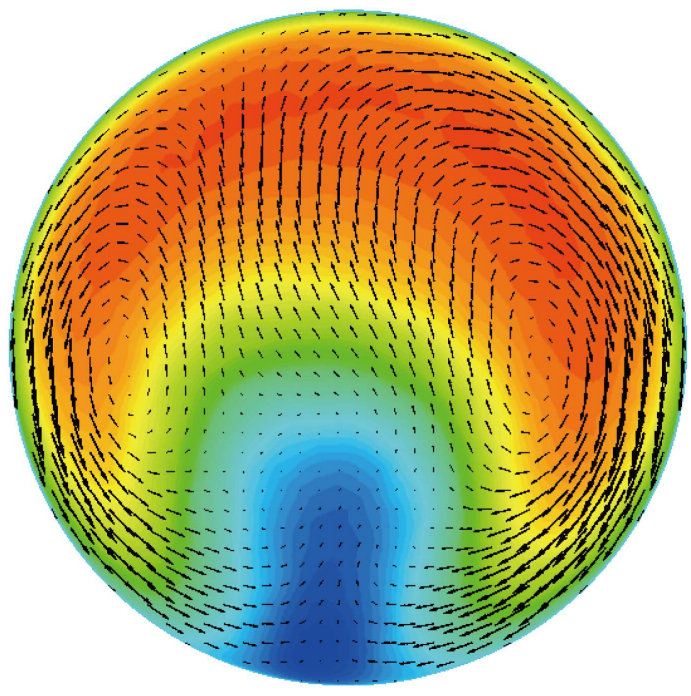

(b) 


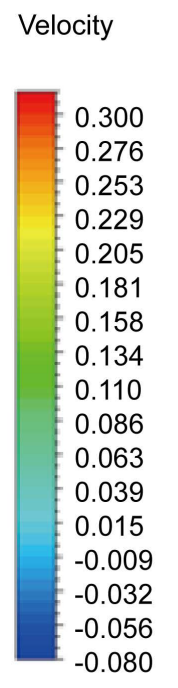

$\left[\mathrm{m} \mathrm{s}^{\wedge}-1\right]$

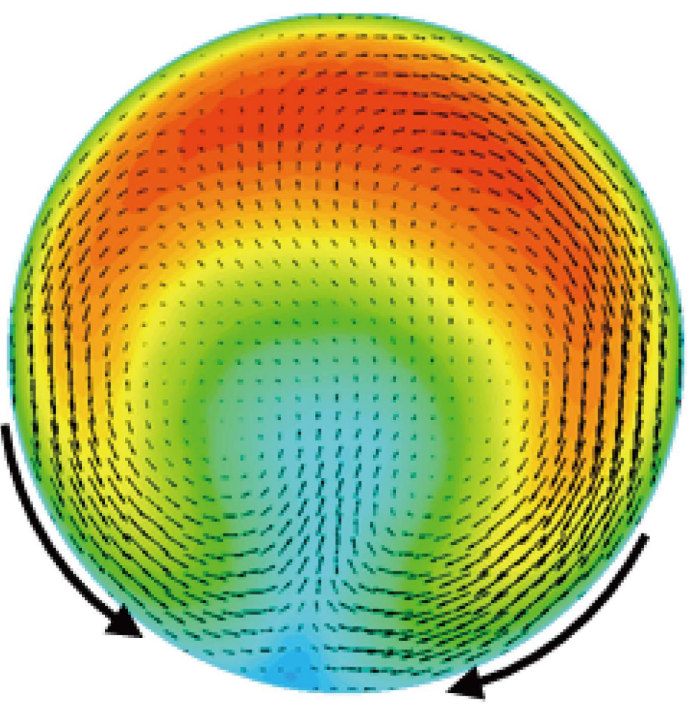

(c)
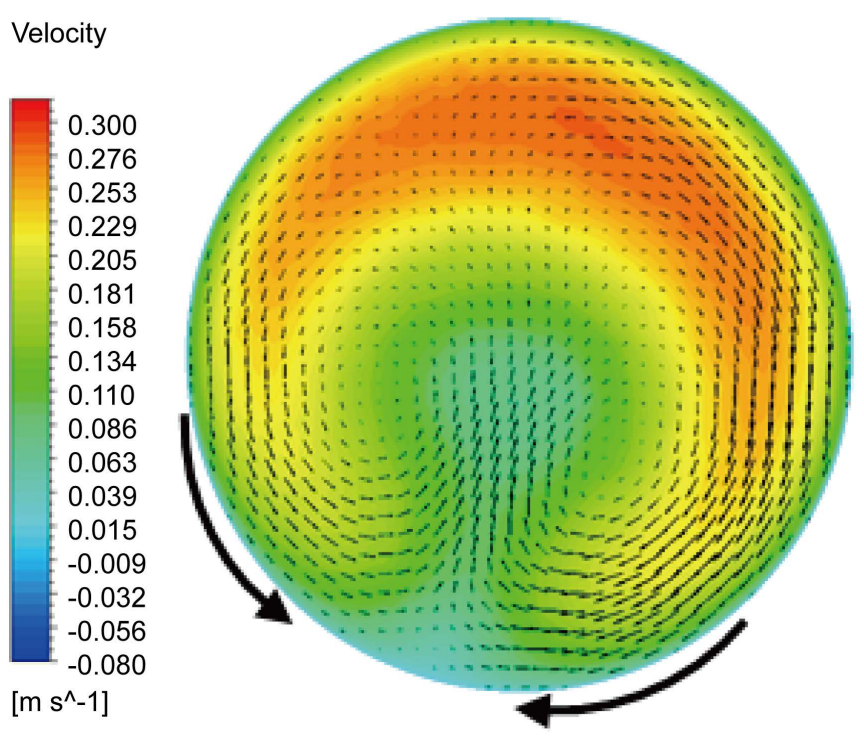

(d)

Figure 11. Cross-sectional velocity distributions of swirling flow downstream of the third elbow. (a) $x / D=0.2$; (b) $x / D=0.6$; (c) $x / D$ $=1 ;(\mathrm{d}) x / D=1.5$.

and reverse flow is no longer observed. Furthermore, the secondary flow pattern is non-symmetric. This result is deviated from that of the velocity field downstream of a single elbow, where symmetrical secondary flow patterns are observed in the cross-section of the pipe [13]. The magnitude of axial velocity in the outer layer decreases and that in the inner layer increases in the triple elbow layout, which indicates the recovery of the highly distorted velocity profile downstream. It should be mentioned that the non-axisymmetry of the cross-sectional velocity distribution can be caused by the influence of the inlet swirling flow condition [7]. 


\section{Conclusion}

Experimental and numerical investigations were carried out to understand the flow structure and velocity field in a triple elbow pipe layout under an inlet swirling flow condition. The two-dimensional velocity field was measured using the phased array UVP system, which allowed the confirmation of flow separation and reattachment in the flow field. The flow separation occurs around the edge of the sharp elbow of the intrados, and the reattachment point is located near $x / D=1$. The numerical simulation shows that the swirling flow structure is quite similar to the experimental result except for a minor difference in the reverse flow region near the separation point. Thus, the CFD prediction was validated by the experimental data. In the case of cross-sectional visualization in the numerical simulation, two counter-rotating vortices were observed between $x / D$ $=0.2$ and 0.6. The two vortex cells are removed from the cross-sectional flow pattern downstream. It should be mentioned that non-axisymmetry of cross-sectional velocity distribution is observed downstream of the triple elbow pipe layout, owing to the influence of the inlet swirling flow condition.

\section{Conflicts of Interest}

The authors declare no conflicts of interest regarding the publication of this paper.

\section{References}

[1] Ono, A., Kimura, N., Kamide, H. and Tobita, A. (2011) Influence of Elbow Curvature on Flow Structure at Elbow Outlet under High Reynolds Number Condition. Nuclear Engineering and Design, 241, 4409-4419. https://doi.org/10.1016/j.nucengdes.2010.09.026

[2] Yuki, K., Hasegawa, S., Sato, T., Hashizume, H., Aizawa, K. and Yamano, H. (2011) Matched Refractive-Index PIV Visualization of Complex Flow Structure in a Three Dimensionally Connected Dual Elbow. Nuclear Engineering and Design, 241, 4544-4550. https://doi.org/10.1016/j.nucengdes.2010.12.026

[3] Kim, J., Yadav, M. and Kim, S. (2014) Characteristics of Secondary Flow Induced by 90-Degree Elbow in Turbulent Pipe Flow. Engineering Applications of Computational Fluid Mechanics, 8, 229-239. https://doi.org/10.1080/19942060.2014.11015509

[4] Mizutani, J., Ebara, S. and Hashizume, H. (2016) Evaluation of the Influence of the Inlet Swirling Flow on the Flow Field in a Triple Elbow System. International Journal of Hydrogen Energy, 41, 7233-7238. https://doi.org/10.1016/j.ijhydene.2016.02.040

[5] NISA (2005) Secondary Piping Rupture Accident at Mihama Power Station, Unit 3, of the Kansai Electric Power Co. Inc. (Final Report). http://www.atomdb.jnes.go.jp/content/000025568.pdf

[6] Fujisawa, N., Yamagata, T., Kanno, S., Ito, A. and Takano, T. (2012) The Mechanism of Asymmetric Pipe-Wall Thinning behind an Orifice by Combined Effect of Swirling Flow and Orifice Bias. Nuclear Engineering and Design, 252, 19-26. https://doi.org/10.1016/j.nucengdes.2012.07.001

[7] Takano, T., Ikarashi, Y., Uchiyama, K., Yamagata, T. and Fujisawa, N. (2016) In- 
fluence of Swirling Flow on Mass and Momentum Transfer Downstream of a Pipe with Elbow and Orifice. International Journal of Heat and Mass Transfer, 92, 394-402. https://doi.org/10.1016/j.ijheatmasstransfer.2015.08.087

[8] Ebara, S., Sato, T., Hashizume, H., Aizawa, K. and Yamano, H. (2010) PIV Measurement for Dual Elbow Flow Using 1/7-Scale Model of Cold-Leg Piping in a Sodium-Cooled Fast Reactor. Proc. 7 th International Conference on Flow Dynamics, Sendai, 1-3 November 2010, 108-109.

[9] Ebara, S., Takamura, H., Hashizume, H. and Yamano, H. (2016) Characteristics of Flow Field and Pressure Fluctuation in Complex Turbulent Flow in the Third Elbow of a Triple Elbow Piping with Small Curvature Radius in Three-Dimensional Layout. International Journal of Hydrogen Energy, 41, 7139-7145.

https://doi.org/10.1016/j.ijhydene.2016.02.068

[10] Vester, A.K., Örlü, R. and Alfredsson, P.H. (2016) Turbulent Flows in Curved Pipes: Recent Advances in Experiments and Simulations. Applied Mechanics Reviews, 68, Article ID: 050802. https://doi.org/10.1115/1.4034135

[11] Al-Rafai, W.N., Tridimas, Y.D. and Woolley, N.H. (1990) A Study of Turbulent Flows in Pipe Bends. Proceedings of the Institution of Mechanical Engineers, Part C: Journal of Mechanical Engineering Science, 204, 399-408. https://doi.org/10.1243/PIME_PROC_1990_204_120_02

[12] Hilgenstock, A. and Ernst, R. (1996) Analysis of Installation Effects by Means of Computational Fluid Dynamics-CFD versus Experiments? Flow Measurement and Instrumentation, 7, 161-171. https://doi.org/10.1016/S0955-5986(97)88066-1

[13] Kalpakli, A. and Örlü, R. (2013) Turbulent Pipe Flow Downstream a $90^{\circ}$ Pipe Bend with and without Superimposed Swirl. International Journal of Heat and Fluid Flow, 41, 103-111. https://doi.org/10.1016/j.ijheatfluidflow.2013.01.003

[14] Chang, T.H. and Lee, H.S., (2003) An Experimental Study on Swirling Flow in a 90 Degree Circular Tube by Using Particle Image Velocimetry. Journal of Visualization, 6, 343-352. https://doi.org/10.1007/BF03181741

[15] Takeda, Y. (1986) Velocity Profile Measurement by Ultrasound Doppler Shift Method. International Journal of Heat and Fluid Flow, 7, 313-318. https://doi.org/10.1016/0142-727X(86)90011-1

[16] Takeda, Y. and Kikura, H. (2002) Flow Mapping of the Mercury Flow. Experiments in Fluids, 32, 161-169. https://doi.org/10.1007/s003480100296

[17] Fukumoto, T., Tsukada, K., Ihara, T., Tsuzuki, N. and Kikura, H. (2013) A Study of Phased Array Ultrasonic Velocity Profile Monitor for Flow Rate Measurement. Proc. 21 st International Conference on Nuclear Engineering, Chengdu, 29 July-2 August 2013, Paper No. ICONE21-16601, 1-6. https://doi.org/10.1115/ICONE21-16601

[18] Örlü, R. (2009) Experimental Studies in Jet Flows and Zero Pressure-Gradient Turbulent Boundary Layers. Doctoral Dissertation, Royal Institute of Technology KTH Mechanics, Stockholm.

[19] A. F. U. Guide (2011) Release 14.0. ANSYS Inc., Canonsburg. 


\section{Nomenclature}

a: single element width [mm]

c. sound speed in water $[\mathrm{m} / \mathrm{s}]$

$d$ : inter-element spacing [mm]

I: piezoelectric element length [mm]

$k$. turbulence kinetic energy $[\mathrm{J} / \mathrm{kg}]$

w: piezoelectric element width [mm]

$D$ : pipe diameter $[\mathrm{mm}]$

$r$. radial distance from a pipe axis $[\mathrm{mm}]$

$R$ : radius of the pipe curvature [mm]

$S$ : swirl intensity, Equation (5) [-]

$U$ : bulk velocity $[\mathrm{mm} / \mathrm{s}]$

Re: Reynolds number $(=U D / v)[-]$

$f_{D i}$ : Doppler shift frequency, which is observed at $\mathrm{i}^{\text {th }}$ channel elements $[\mathrm{Hz}]$

$f_{\dot{0}}$ : basic frequency of phased array sensor $[\mathrm{Hz}]$

$f_{p r i}$ pulse repetition frequency $[\mathrm{kHz}]$

$N_{\text {rep}}$ : number of repetitions [-]

$\boldsymbol{e}_{e}$ : unit vector in the direction of measurement line [-]

$\boldsymbol{e}_{i}$ : unit vector in the direction from the particle to $\mathrm{i}^{\text {th }}$ channel elements [-]

$V$ : velocity vector of the moving particle $[\mathrm{mm} / \mathrm{s}]$

$\theta_{s}$ : phased steering angles [degree]

$\gamma$ : pipe diameter to elbow radius ratio $[=D / R][-]$

$\omega$ : angular velocity of swirl generator $[1 / \mathrm{min}]$

$\varepsilon$ : turbulence dissipation rate $\left[\mathrm{m}^{2} / \mathrm{s}^{3}\right]$

$\Delta t$. time delay of ultrasound pulse [s]

$\Delta y$ : spatial resolution $[\mathrm{mm}]$

Subscripts

$r, \theta$ : cylindrical coordinates

$x, y$. axial coordinates 